\title{
Bandgaps of Atomically Precise Graphene Nanoribbons and Occam's Razor
}

\author{
Aristides D. Zdetsis* \\ Molecular Engineering Laboratory, Department of Physics, University of Patras, Patras 26500 \\ GR, Greece.
}

\begin{abstract}
Rationalization of energy gaps of atomically precise AGNRs, "bulk" $\left(\Delta \mathrm{E}_{\mathrm{ac}}\right)$ or "zigzag-end" $\left(\Delta \mathrm{E}_{\mathrm{zz}}\right)$, could be challenging and controversial concerning their magnitude, origin, substrate influence $\left(\Delta \mathrm{E}_{\mathrm{sb}}\right)$, and spin-polarization, among others. Hereby, a simple self-consistent and "economical" interpretation is presented, based on "appropriate" DFT (and TDDFT) calculations, general symmetry principles, and plausibility arguments, which is fully consistent with current experimental measurements for 5-, 7-, and 9-AGNRs within less than 1\%, although at variance with some prevailing views or interpretations for $\Delta \mathrm{E}_{\mathrm{ac}}, \Delta \mathrm{E}_{\mathrm{zz}}$, and $\Delta \mathrm{E}_{\mathrm{sb}}$. Thus, an excellent agreement between experiment and theory emerges, provided some established stereotypes are reconsidered and/or abandoned. The primary source of discrepancies is the finite length of AGNRs together with inversion-symmetry conflict and topological end/edge states, which invariably mix with other "bulk" states making their unambiguous detection/distinction difficult. This can be further tested by eliminating end-states (and $\Delta \mathrm{E}_{\mathrm{zz}}$ ), by eliminating empty (non-aromatic) end-rings
\end{abstract}

Keywords: Armchair Graphene Nanoribbons (AGNRs), Atomically Precise AGNRs, "bulk"/“surface” Energy Gaps, Topological end-states

*zdetsis@upatras.gr 


\section{TOC Graphic}

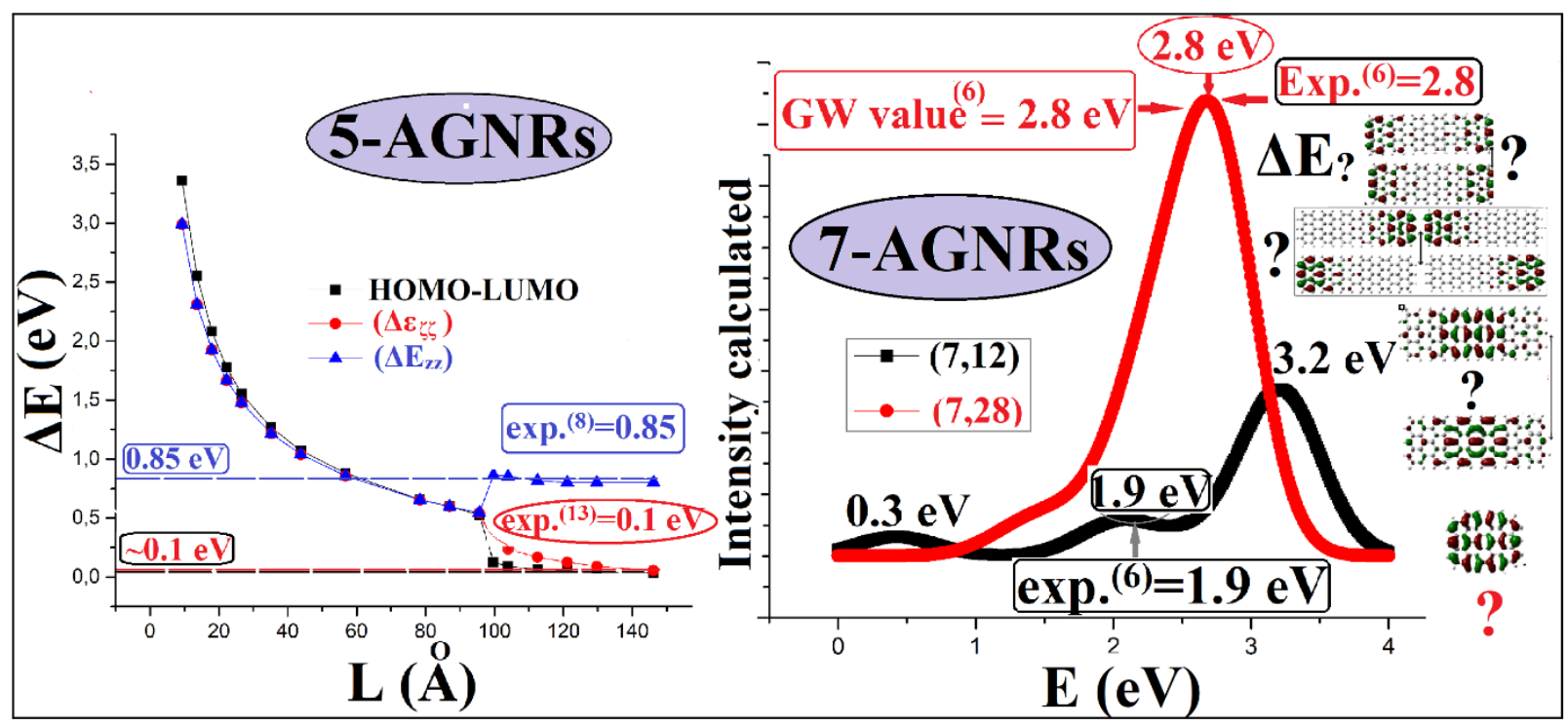

\section{Highlights}

- Excellent agreement $< \pm 1 \%$ with measured energy gaps of 5, 7, 9-AGNRs is achieved.

- Topological end-states, finite length and inversion symmetry conflict are vital.

- These results imply marginal substrate contribution to the measured STS gaps,

- maximum Coulomb correlation of almost equal magnitude with the staggered potential,

- Closed singlet ground state with possibly non-conventional magnetism. 
1. Introduction Edge or end states in graphene nanoribbons (GNRs), and in particular armchair GNRs (AGNRs) have attracted very much interest lately, ${ }^{1-8}$ due to their anticipated magnetic properties, ${ }^{9-}$ ${ }^{10}$ although their presence in finite nanographenes (NGRs) has been predicted long time ago. ${ }^{11}$ However, the significance and importance of end states for AGNRs was recognized only recently ${ }^{1-}$ ${ }^{8}$, after the pioneering bottom-up synthesis of atomically precise AGNRs of finite lengths L with short zigzag ends ${ }^{3-16}$. Clearly no end states appear in the common infinite AGNRs fabricated by the usual top-bottom techniques, which are theoretically described by periodic boundary conditions at their two ends ${ }^{1}$. The new developments have brought to the forefront new concepts and properties such as the "bulk band gaps" $\Delta \mathrm{E}_{\mathrm{ac}}$ (or $\Delta_{\mathrm{ac}}{ }^{1,6}$ ) i.e., the energy gaps between delocalized states, and the energy separation of the zigzag-end-localized "end-states", denoted here by $\left(\Delta \mathrm{E}_{\mathrm{zz}}\right)\left(\right.$ or $\left.\Delta_{\mathrm{zz}}{ }^{6-8}\right)$, thus increasing both quantity and quality of key properties to be rationalized, understood, or interrelated at the atomic scale. At the same time, despite the increased complexity, such advances have also allowed the study of the L-dependence of key-quantities such as the bandgaps ${ }^{4-7}$ (both, $\Delta \mathrm{E}_{\mathrm{ac}}$ and $\Delta \mathrm{E}_{\mathrm{zz}}$ ), conductivity, aromaticity ${ }^{1,3-4}$, and even Raman spectra. ${ }^{17}$ The L-dependence studies $^{4-5}$ revealed that the changes in such properties versus length are not gradual (or smooth). The presence of a phase transition at a critical length $\mathrm{L}_{\mathrm{c}}$ was advocated by two different recent works, Lawrence et al. ${ }^{8}$ and Zdetsis et al. ${ }^{4}$, almost simultaneously. However, the two works have offered different assessments and interpretations for the nature of the transition and magnetism, as well as the value of $\mathrm{L}_{c} .{ }^{4,8}$ This is not something new or unusual in a rapidly grown pioneering field like this ${ }^{1}$, and this is not the only existing "discrepancy". Other conflicting (or conflicting-looking) results (experimental and theoretical) include the magnitude and nature of the bandgaps ${ }^{1,6-7,12-13}$, the existence and nature of magnetism in the edge states $^{1,3-7}$, as well as the magnitude of the substrate influence on these properties. ${ }^{1,5-7}$ For example, the magnitude of the bandgap for the 5AGNRs has been measured by (at least) three different groups ${ }^{8,12-13}$ to be $0.85 \mathrm{eV}^{8}, 2.8 \mathrm{eV}^{12}$, and 
$0.1 \mathrm{eV}^{13}$ respectively, while the theoretical values vary from $0.1 \mathrm{eV}^{1}$ to $1.7 \mathrm{eV} .{ }^{14}$ For the 7-AGNRs the measured values of $\Delta \mathrm{E}_{\mathrm{zz}}$ vary between $1.9 \mathrm{eV}^{6}$ and $2.5 \mathrm{eV}^{7}$, whereas the measured $\Delta \mathrm{E}_{\mathrm{ac}}$ values range from $2.3 \mathrm{eV}$ to $3.2 \mathrm{eV}^{6-7,9,15}$, overlapping significantly with the range of $\Delta \mathrm{E}_{\mathrm{zz}}$. Thus, the unambiguous distinction between $\Delta \mathrm{E}_{\mathrm{ac}}$ and $\Delta \mathrm{E}_{\mathrm{zz}}$ is another subtle point together with the bridging of the measured and calculated $\Delta \mathrm{E}_{\mathrm{ac}}$ values, which also vary widely from $2.3 \mathrm{eV}$ to $3.7 \mathrm{eV} .1,6,7,14$ Some of the (different) measured or calculated values correspond to AGNRs of different length, but in the literature the quoted values are usually given without reference to the actual length which is, thus, treated as a hidden variable. However, the biggest problem seems to be the large difference between the measured values of the gap(s) in relation to the "official" theoretical values obtained by the GW method ${ }^{14}$, which are widely recognized as an almost universal point of reference. Such large differences (almost $\sim 1.5 \mathrm{eV}$ for the 7-AGNRs) between experimental and theoretical GW gaps $\left(\Delta \mathrm{E}_{\mathrm{ac}}\right)$ are usually attributed to the screening from the metallic (Au) substrate $\Delta \mathrm{E}_{\mathrm{sb}}$, even though identical values of gap (within the experimental uncertainties) have been obtained for AGNRs grown on non-metallic substrates, such as $\mathrm{NaCl}^{6}$ and $\mathrm{MgO}^{7}$. This is clearly (at least) problematic. All these subtle points, including also the confusion in distinguishing between $\Delta \mathrm{E}_{\mathrm{ac}}$ and $\Delta \mathrm{E}_{\mathrm{zz}}$ gaps, need new and further investigation and interpretation(s). This is the target of the present work, which can be considered as a positive synthesis of various conflicting views. Based on previous experience, ${ }^{1,3,19}$ it is expected that such synthesis should be proven successful and constructive, facilitating the successful and accurate functionalization of AGNRs for realistic applications. As is demonstrated below, we can fully rationalize all known experimental data for the 5-, 7-, and 9-AGNRs within less than $1 \%$ accuracy, and pinpoint at the same time the sources of discrepancies.

2. Theoretical framework. For a consistent and transparent understanding and interpretation of the origin and magnitude of $\Delta \mathrm{E}_{\mathrm{ac}}, \Delta \mathrm{E}_{\mathrm{zz}}$ as well as the factors that influence their size, it is important to realize that practically all these quantities are dominated by the influence of the ("many-body") 
Coulomb correlation energy combined with the sublattice frustration, which gives rise to the staggered sublattice potential ${ }^{20}$ across the zigzag ends of finite length AGNRs (or the zigzag edges of ZGNRs). In fact, the sublattice frustration, which is the driving force for the generation of the end/edge states, as we have illustrated earlier, ${ }^{2-4}$ constitutes the largest (or even the full) contribution on the Coulomb correlation energy. The understanding that most (or all) of the Coulomb correlation energy is devoted to counterbalance the topological frustration between sublattice and molecular symmetry-groups is the starting (and key) point of the present investigation. This principle together with the established ${ }^{2-5}$ (hidden) strong contributions of aromaticity and shell structure ${ }^{2-5}$ constitute the basis for the deeper understanding of all these quantities ( $\Delta \mathrm{E}_{\mathrm{ac}}, \Delta \mathrm{E}_{\mathrm{zz}}, \Delta \varepsilon_{\zeta \zeta}$, and $\left.\Delta \mathrm{E}_{\mathrm{s}}\right)$. Thus, if we can properly alleviate the sublattice-molecular group symmetry frustration (which is equivalent with inversion symmetry conflict), under the natural constrains of shell structure and aromaticity, we could effectively account for the (largest part of) Coulomb correlation energy. This is compatible with the conclusions of Ijäs et al. ${ }^{21}$ that the single particle description used in DFT studies, as the present one, is justified for graphene $\pi$ states.

2.1 Calculation of $\Delta \mathbf{E}_{\mathbf{z z}}$ and $\Delta \mathbf{E}_{\mathbf{a c}}$. Within the 1-electron approximation underlining the DFT and HF self-consistent fields, the symmetry frustration between molecular $\left(\mathrm{D}_{2 \mathrm{~h}}\right)$ and sublattice $\left(\mathrm{C}_{2 \mathrm{~V}}\right)$ symmetry groups can be alleviated by effectively breaking (or redefining) the symmetry of the additional degrees of freedom (besides spatial coordinates) i.e., the spin and/or pseudospin (for real-space calculations). In the first case we can introduce non-zero spin values preserving the molecular symmetry, ${ }^{3}$ whereas in the second case we are forced to break molecular symmetry, by introducing open-shell singlet states, which when optimized geometrically converge normally to $\mathrm{C}_{2 \mathrm{v}}$ symmetric geometries compatible with sublattice symmetry, thus breaking the molecular symmetry as well. This is illustrated in Fig. $1(\mathrm{a}, \mathrm{b})$. The molecular D2h symmetry demands same type (same sublattice) atoms at the two ends, as shown in Fig. 1(a), whereas the sublattice C2v symmetry requires opposite type atoms. 


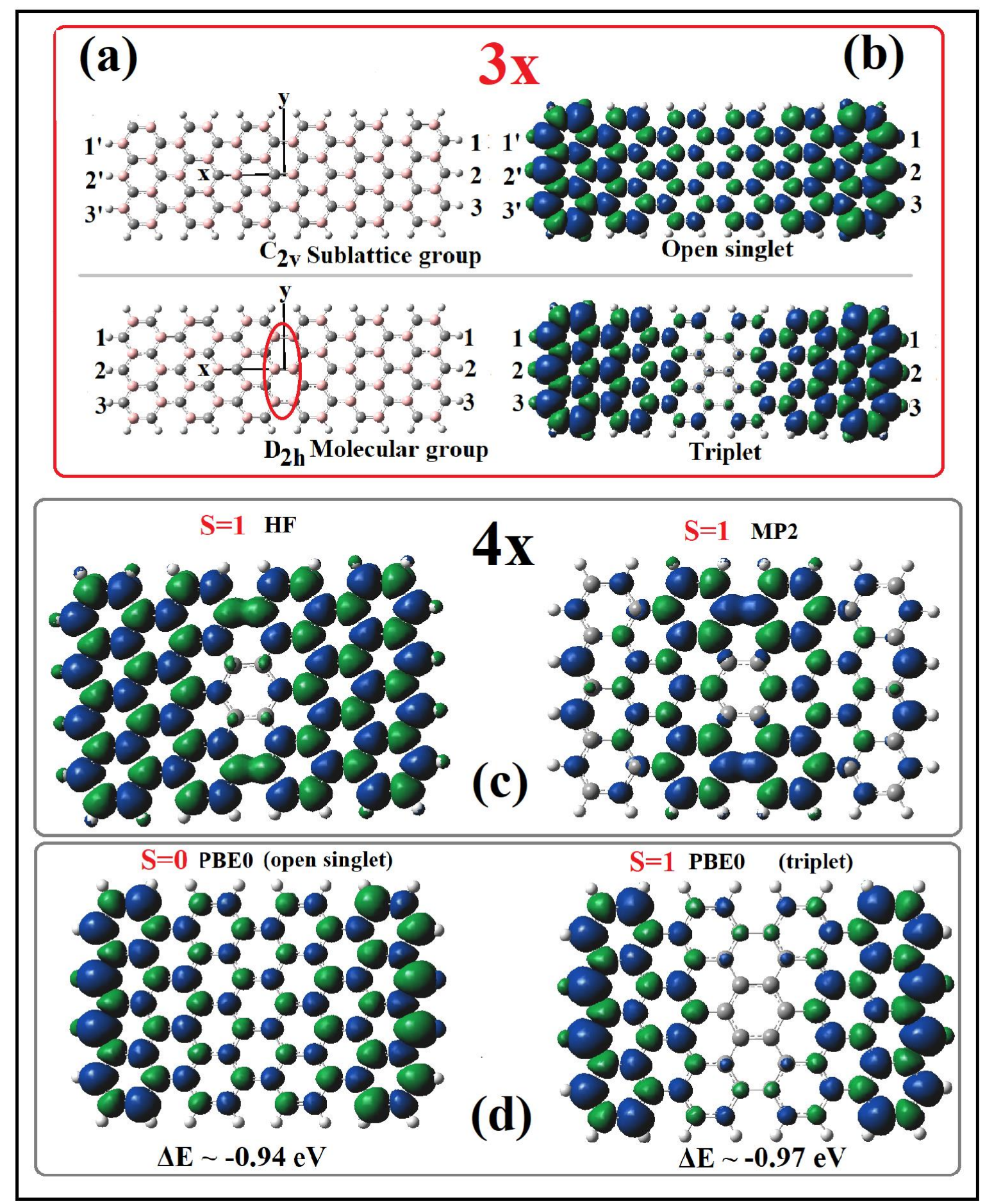

FIGURE 1. Molecular and sublattice symmetry of the 3x6 $(7,12)$ AGNR (a), reflected in the spin densities (b). The vertical elliptic curve indicates the region of sublattice imbalance (and frustration). Comparison of the corresponding spin densities for the $4 \times 4(9,8)$ AGNR at the HF, MP2 and DFT/PBE0 level is given in (c) and (d). 
This is reproduced in the corresponding "spin" densities (b), which for open singlet reflects the sublattice symmetry (with different type atoms at the two ends), while the triplet state for the same reason has a region of zero spin in the middle, exactly where the sublattice imbalance occurs. In the ordinary "atomistic" calculations the sublattice degree of freedom does not enter in the spatial Hamiltonian and can only be introduced as (pseudo) spin. Then, due to the better account of Coulomb interaction, open shell states (triplet or singlet) appear energetically lower than the closed singlet state. This is because the additional degree of freedom of "pseudospin", introduced to take care of the sublattice topology (and the staggered potential), facilitates the optimization of Coulomb interaction by keeping away of each other electrons of different spin (for which Pauli repulsion is not operative), but identical pseudospins. Moreover, based on the shell model ${ }^{2-3}$, the unoccupied states of the "previous" (shell number smaller by 1) AGNR are the occupied of the current AGNR. This is responsible for the interplay between odd and even parity HOMOs as the width of AGNRs is growing (3n AGNRs have odd HOMO and even LUMO, whereas 3n+1 AGNRs are characterized by even HOMO and odd LUMO). ${ }^{2-3}$ This is also responsible for the well-known $3 n, 3 n \pm 1$ width rule for AGNRs. ${ }^{3}$ Note that the (pseudo)spin densities invariably reflect the sublattice (pseudospin) structure within the frustrated molecular $\left(\mathrm{D}_{2 \mathrm{~h}}\right)$ symmetry $^{3}$ in the first case, or the sublattice symmetry $\left(\mathrm{C}_{2 \mathrm{v}}\right)$ in the latter (see Fig. S1), where opposite end sites have opposite spins. In both cases the central region is characterized by almost zero (pseudo)spin. It should be emphasized at this point that for wider AGNRs (where $n>1$ in the above width rule ${ }^{3}$ ), higher spin states are required $^{3}$ to lower the total energy (within the molecular $\mathrm{D}_{2 \mathrm{~h}}$ symmetry group). Such larger (pseudo) spin-polarized states optimize better the sublattice distribution (within the $\mathrm{D}_{2 \mathrm{~h}}$ molecular group), ${ }^{3}$ whereas the open-shell singlets lye higher in energy and revert to the closed singlet state. This illustrates emphatically that the open-singlet state is not the true ground (lowest energy) state of AGNRs (and, consequently, no conventional magnetism is truly present). Nevertheless, the open singlet state is still a very useful and efficient concept for the description of end-states, as is 
illustrated below. It should be emphasized that in both cases of Fig. 1, when correlation is introduced even at the MP2 level, the energetical ordering is reversed and the lowest energy structure is a closed singlet. ${ }^{4}$ In addition, the MP2 correlated "spin" density of the triplet, as we can see in Fig. 1 (c), is rather correcting the HF failure (having the opposite sign) than reflecting the full sublattice structure. Note also in Fig. $1(\mathrm{c}, \mathrm{d})$ that the triplet state is slightly lower than the open singlet, and that the energy difference of the open shell singlet and triplet states (which are practically isoenergetic) from the closed singlet is about $0.95 \mathrm{eV}$. This should be a good estimate of the "missing" Coulomb energy in this case, and, based on the (approximate) electron-hole symmetry, the expected (HOMO-LUMO) separation of the open singlet (or the triplet) should be about twice as large $(\sim 2 \mathrm{eV})$. Indeed, the calculated open-singlet HOMO-LUMO gap for the 9AGNRS (or $4 \mathrm{x}$ ) is $2.2 \mathrm{eV}$, and so is $\Delta \mathrm{E}_{\mathrm{zz}}$ (vide infra). Even more important is the fact that the corresponding value for the $3 \times 6$ or $(7,12)$ AGNR is also about $0.93 \mathrm{eV}$, suggesting an open-singlet gap of about 1.9 which is in excellent agreement with both the measured value ${ }^{6}$ of $\Delta \mathrm{E}_{\mathrm{zz}}(1.90 \mathrm{eV})$, and the calculated open singlet gap. It is important to observe also that the open-singlet value " $\Delta \mathrm{E}_{\mathrm{zz}}$ " $=1.2 \mathrm{eV}$ for the 5-AGNRs and the $1.9 \mathrm{eV}$ open singlet gap for the 7-AGNRs are practically equal to the correlation improved GW-LDA bandgap differences, ${ }^{14}$ which is highly suggestive for the essential correctness of our claim. Thus, within the one-electron approximation we have established the correct basis for discussion and analysis of both $\Delta \mathrm{E}_{\mathrm{zz}}$ and $\Delta \mathrm{E}_{\mathrm{ac} .} \Delta \mathrm{E}_{\mathrm{zz}}$ is identified as the opensinglet HOMO-LUMO gap, whereas $\Delta \mathrm{E}_{\mathrm{ac}}$ can be identified as the difference |(HOMO-1)$(\mathrm{LUMO}+1)$, with the understanding that both HOMO and LUMO are end-states. It should be emphasized however that the central meaning of $\Delta \mathrm{E}_{\mathrm{zz}}$ is only valid for lengths $\mathrm{L}$ longer than the critical length ( $\mathrm{L} \geq \mathrm{L}_{\mathrm{c}}$ ), although the open-singlet HOMO-LUMO is defined for almost all lengths and is practically constant, as is verified also by Wang et al. ${ }^{6}$ For both gaps ( $\Delta \mathrm{E}_{\mathrm{ac}}$ and $\Delta \mathrm{E}_{\mathrm{zz}}$ ) we can further correct if we wish their (one-body) values by considering additional many body contributions through time-dependent DFT (TDDFT), which has been shown ${ }^{1}$ to provide very good 
("many-body") estimates of the gaps, so that the STS spectrum overall looks very much alike the (luminous) optical spectrum, because both are dominated by molecular overlaps between transition states. This is further illustrated and "verified" from the results below. Furthermore, the use of TDDFT allows the clear and unambiguous identification of the energy separation of the end/edge states, which according to the present investigation is not given by $\Delta \mathrm{E}_{\mathrm{zz}}$, as Wang et $a .^{6}{ }^{6}$ have suggested, but by another type of gap which here is denoted as $\Delta \varepsilon_{\zeta \zeta}$. In the usual one-body approximation $\Delta \varepsilon_{\zeta \zeta}$ corresponds to the HOMO-LUMO separation of the closed singlet true ground state for $\mathrm{L} \geq \mathrm{L}_{\mathrm{c}}$, which is always only a few $0.1 \mathrm{eV}(\sim 0.1 \mathrm{eV}$, for $\mathrm{L} \rightarrow \infty)$ in accord with the association of the end states with the Dirac points ${ }^{3-4}$ (and charge neutrality points ${ }^{4}$ ) located "very close" to the fermi level. TDDFT indeed verifies that in contrast to $\Delta \varepsilon_{\zeta \zeta}$ which involves transition from one purely end-localized HOMO state to an opposite-parity end-localized LUMO state, $\Delta \mathrm{E}_{\mathrm{zz}}$ gap always involves transitions from a mixture $(\sim 60 \%-\sim 40 \%)$ of "surface"-"bulk" states to another state of about equal amount of mixing. Thus, although $\Delta \mathrm{E}_{\mathrm{zz}}$ involves a large amount of localized end-states, it should not be associated with the energy separation of the end-states. Another way, besides TDDFT, to distinguish between "bulk" and "surface" energy gaps is by comparing to the corresponding "edge-modified" AGNRs, ${ }^{5}$ obtained by eliminating "empty" (i.e., non-aromatic) end-rings, which also eliminates topological end-states (and, therefore, $\Delta \mathrm{E}_{\mathrm{zz}}$ and $\Delta \varepsilon_{\zeta \zeta}$ ).

2.2 The Substrate influence on the measured STS gaps. As we have mentioned above, the main crucial property under possible dispute is the magnitude of the substrate influence (screening) $\Delta \mathrm{E}_{\mathrm{sb}}$ on the measured STS gap. According to our earlier estimates ${ }^{1} \Delta \mathrm{E}_{\mathrm{sb}}$ should be of the order of a few $0.1 \mathrm{eV}$. However, almost in all cases $\Delta \mathrm{E}_{\mathrm{sb}}$ larger than $1 \mathrm{eV}$ is needed to bridge the experimental STS measurements for AGNRs deposited on metal surfaces (usually Au) and the theoretical values for free standing AGNRs. The theoretical values widely recognized as an almost universal point of reference are the GW results of Yang et al.,${ }^{14}$ which among the theoretical values reported earlier are clearly the largest, and many times by far. As a result, $\Delta \mathrm{E}_{\mathrm{sb}}$ which is defined as 
the difference of the STS measurements and the theoretical reference values are unrealistically large. For example, for the 7-AGNRs the theoretical GW gap ${ }^{14}$ is $3.7 \mathrm{eV}$, whereas the experimental STS gap value obtained by various groups ${ }^{6-7,9,15}$ is $2.5 \pm 0.2 \mathrm{eV}$. Thus, $\Delta \mathrm{E}_{\mathrm{sb}}$ should be at least 1.2 $\mathrm{eV}$. However, the STS value of $2.5 \mathrm{eV}$ was also obtained for 7-AGNRs deposited on non-metallic substrates, such as $\mathrm{NaCl}^{6}$ and $\mathrm{MgO}^{7}$, for which such large $\Delta \mathrm{E}_{\mathrm{sb}}$ value is clearly unrealistic. On the basis of their STS measurements on samples grown on $\mathrm{MgO}$, Kolmer et al. ${ }^{7}$ concluded that $\Delta \mathrm{E}_{\mathrm{sb}}$ should be marginal, which is in full agreement with our present results. However, the general consensus, with few exceptions ${ }^{1,7,18}$ is largely different (up to now). In this work we are led to conclude that the GW results ${ }^{14}$ overestimate the bandgaps mainly due to the size effect, since the GW results of Yang et al. ${ }^{14}$ were obtained for infinite AGNRs, whereas the atomically precise AGNRs have finite length (and topological end-states). This could be sufficient to explain the resulting unrealistically large $\Delta \mathrm{E}_{\mathrm{sb}}$ values. Yet, besides the infinite size (and the corresponding periodic boundary conditions) the lack of exact exchange in the LGA wavefunctions building the Green's function could be also important since exchange interaction is very sensitive to inversion symmetry frustration. Nevertheless, judging from our TDDFT results, it is more reasonable to attribute the gap difference between the infinite and the finite size AGNRs (size effect) to the mixing of edge/end states with the infinite "bulk" states (and the scattering at the zigzag edges) which can drastically reduce the gap. This is corroborated by the GW results ${ }^{6}$ of Wang et al. ${ }^{6}$ for the finite (7, 24) AGNR (and slightly longer), who obtained a gap of $2.8 \mathrm{eV}$ clearly closer to the measured (by several groups) gap, and substantially smaller compared to the $3.7 \mathrm{eV}\left(\mathrm{G}_{0} \mathrm{~W}_{0}\right)$ value ${ }^{14}$ for the infinite 7-AGNR. Parenthetically, it should be mentioned at this point that even in the worst-case scenario where the substrate interaction is strong (especially when the distance of STS tip from the surface is small), this leads to mixed substrate-AGNR states ${ }^{1}$ which can be easily recognized (and excluded) from the measurements by comparing the two separate STS spectra. Moreover, such states would be expected to have low overlap with the pure AGNR- excited-states, and consequently the 
corresponding transition(s) would have very low intensity and would be difficult to detect. Thus, we assert here that $\Delta \mathrm{E}_{\mathrm{sb}}$ should indeed be marginal, in full agreement with the experiment al results (for 7-AGNRs grown directly on $\mathrm{MgO}$ substrate) and conclusions of Kolmer et al. ${ }^{7}$.

2.3 Computational details. The theoretical and computational details of the present investigation have been described in references 1 through 5 . The computations, as before, have been performed with the Gaussian ${ }^{22}$ program package, while the visualization of the results was accomplished using the GaussView software. ${ }^{23}$

\section{Results and discussion}

3.1 5-AGNRs. Figure 2 summarizes the present results for the 5-AGNRs (or 2x AGNRs)

which, as mentioned earlier, have been also studied by several groups. ${ }^{1,4,8,12-13,16}$

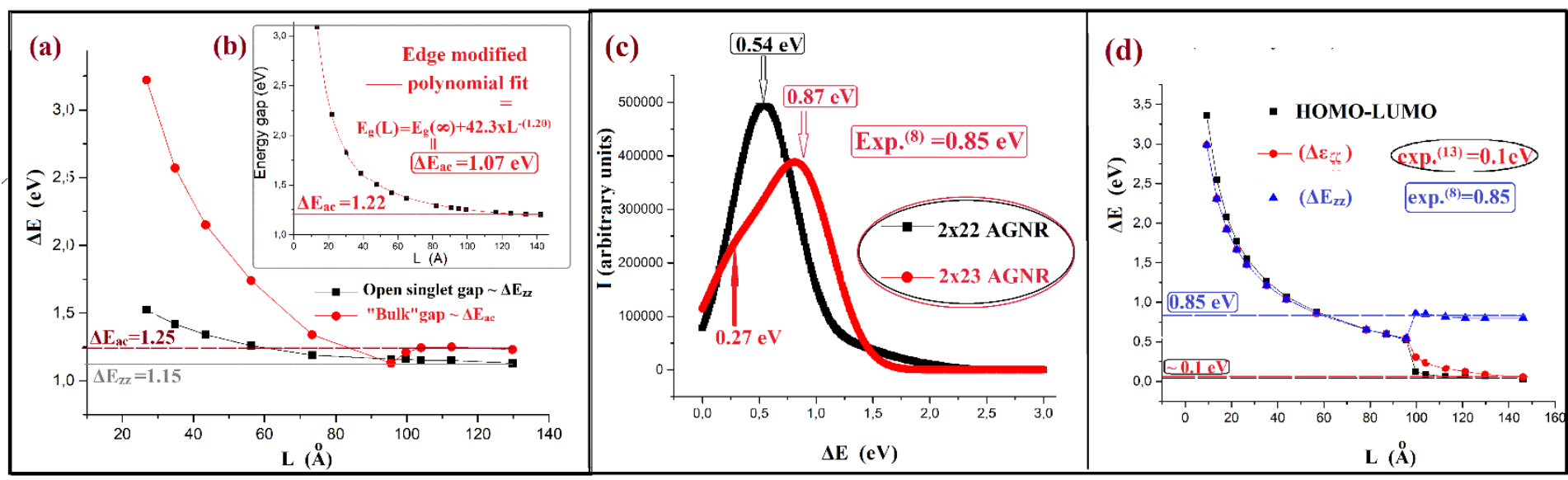

FIGURE 2. (a): Variation of the open singlet, $\Delta \mathrm{E}_{\mathrm{zz}}$, and the "bulk" $|(\mathrm{HOMO}-1)-(\mathrm{LUMO}+1)|=$ $\Delta \mathrm{E}_{\mathrm{ac}}$ gaps (in $\mathrm{eV}$ ) in terms of length $\mathrm{L}$ (in $\AA$ ) for the 5-AGNRs (2x). (b): Variation of $\Delta \mathrm{E}_{\mathrm{ac}}$ gap (in $\mathrm{eV}$ ) as a function of length for the edge modified 5-AGNRs together with the usual polynomial fit (see text). (c): excitation spectrum of the $2 \times 22$ and $2 \times 23$ AGNRs. Intensity (I) is in arbitrary units and excitation energy $(\Delta \mathrm{E})$ in $\mathrm{eV}$. (d): Variation with length of the HOMO-LUMO, and $\Delta \mathrm{E}_{\mathrm{zz}}, \Delta \varepsilon_{\zeta \zeta}$ gaps, calculated by TDDFT as "first" and "second" optical gaps respectively, including the corresponding experimental values from refs. 8, and 13 (see text). 
In Fig.2(a) the open singlet HOMO-LUMO gap, $\Delta \mathrm{E}_{\mathrm{zz}}$, and the "bulk" gap $\Delta \mathrm{E}_{\mathrm{ac}}$ are plotted versus length $\mathrm{L}$. Here, following the discussion above for the open singlet gap and its relation to $\Delta \mathrm{E}_{\mathrm{zz}}$, we have defined $\Delta \mathrm{E}_{\mathrm{zz}}$ as the HOMO-LUMO gap of the open singlet state, contrary to the original definition of Wang et al. ${ }^{6}$ as the energy separation of the end states. Obviously, for an open singlet ground state both definitions are equivalent, but this is not the case. As we can see in Fig. 2(a), the “one-body" $\Delta \mathrm{E}_{\mathrm{ac}}=|(\mathrm{HOMO}-1)-(\mathrm{LUMO}+1)|$ gap after the discontinuity (or transition) at $\mathrm{L} \approx 100 \AA$, which we have discussed in detail in a previous work, ${ }^{4}$ starts opening up at $\mathrm{L}_{\mathrm{c}}$, contrary to the "onebody" $\Delta \mathrm{E}_{z z}$ (i.e., the open singlet HOMO-LUMO gap) which varies slowly and smoothly over the entire range of lengths. This is very strange indeed, if $\Delta \mathrm{E}_{\mathrm{zz}}$ is going to represent the real separation of the edge states, since $\Delta \mathrm{E}_{\mathrm{zz}}$ first appears at and after the transition at $\mathrm{L}_{\mathrm{c}}$. Such behaviour (smooth variation) should be better suited for $\Delta \mathrm{E}_{\mathrm{ac}}$. This is indeed verified in Fig. 2(b), which shows the HOMO-LUMO gap of the "edge modified AGNRs", which seems to saturate to the value of 1.22 $\mathrm{eV}$, very close to the value of $1.25 \mathrm{eV}$, suggested from the behavior of the "normal" AGNRs in Fig. 2(a). The edge modified AGNRs by construction have no edge states and their HOMOs and LUMOs are delocalized over their entire length, ${ }^{4}$ and therefore their fundamental gap corresponds to $\Delta \mathrm{E}_{\mathrm{ac}}$. Such edge-modified AGNRs are obtained by eliminating the empty (non-aromatic) endrings ${ }^{5}$ of the standard AGNRs, which also eliminates end-states and zigzag end-bonds. ${ }^{5}$ This is a clear manifestation of the importance of aromaticity for AGNRs (and graphene itself). ${ }^{2-3}$ Comparing the behavior of the "bulk gap" in Figs. 2(a) and 2(b), we can see that due to quantum confinement (both lateral and longitudinal) the (HOMO-1) and (LUMO+1) states defining the "onebody" $\Delta \mathrm{E}_{\mathrm{ac}}$ are also affected by the abrupt appearance of the edge states, in sharp contrast to the ("one-body") open singlet gap which seems to be practically insensitive to the appearance of the end-states, contrary to what is expected from its original definition. This in fact emphasizes the "many-body" nature of the end states through their connection with inversion symmetry conflict, which is further supported from Figs. 2(c) and S1. The "correct" behavior (with length variation) 
of the "one-body" $\Delta \mathrm{E}_{\mathrm{ac}}$ is given by the (delocalized) HOMO-LUMO gap of the edge-modified AGNRs in Fig. 2(b). As we can see in Fig. 1(b) the value of $\Delta \mathrm{E}_{\mathrm{ac}}$ (HOMO-LUMO gap of the edgemodified AGNRs) as a function of length, as $\mathrm{L} \rightarrow \infty$, seems to saturate to the value of $1.22 \mathrm{eV}$. This could be misleading since only lengths up to about $140 \AA$ A have been considered. To remedy this problem we have recently suggested ${ }^{24}$ to fit the calculated $\Delta \mathrm{E}_{\mathrm{ac}}$ as a function of L efficiently and transparently ${ }^{1,24}$ to a polynomial of the form $\Delta \mathrm{E}_{\mathrm{ac}}(\mathrm{L})=\mathrm{A}+\mathrm{B} \times \mathrm{L}^{-\mathrm{C}}$, where the value $\mathrm{A}$ corresponds to the gap at infinity, $\Delta \mathrm{E}_{\mathrm{ac}}(\infty)=\mathrm{A}$, and the constant $\mathrm{C}$ to some short of effective ("fractal") dimensionality (here equal to 1.20). ${ }^{1,24}$ As we can see in the inset in Fig. 2(b), the projected $\Delta \mathrm{E}_{\mathrm{ac}}$ value is $1.07 \mathrm{eV}$, which is also verified by the TDDFT result $\Delta \mathrm{E}_{\mathrm{ac}}=1.01 \mathrm{eV}$ (see Fig. S1). The TDDFT value $(1.01 \mathrm{eV})$ is clearly closer to the value of $0.85 \mathrm{eV}$ measured by Lawrence et al. ${ }^{8}$, assuming a very reasonable substrate screening (of about $0.15 \mathrm{eV}$ ), as we have suggested recently. ${ }^{4}$ However, further correct information is given in Fig. 2(c), showing the spectra of the $2 \times 22$ and 2x23 AGNRs immediately before and after transition, respectively (see Fig.2(d) too). As is illustrated in Fig. 2(c), in the 2x23 AGNR (immediately after the transition) there is a strong peak value at $0.87 \mathrm{eV}$, very close to the recently measured ${ }^{8}$ STS gap of $0.85 \mathrm{eV}$. Detailed analysis of the TDDFT results shows that this peak includes transitions involving end-states to a large percentage (about $60 \%$ ). Thus, the calculated value of $0.87 \mathrm{eV}$ and the measured ${ }^{8}$ gap should be assigned to $\Delta \mathrm{E}_{\mathrm{zz}}$. This, contrary to the "one-body" gap, restores the expected correct behavior of $\Delta \mathrm{E}_{\mathrm{zz}}$ at (and after) $\mathrm{L}_{\mathrm{c}}$. Even more interesting is the fact that extrapolating to longer AGNRs gives a gap of 0.85 $\mathrm{eV}$ (exactly), which is an unexpected full agreement with experiment, as is shown in Fig. 2(d). Fig. 2(d) also shows that, contrary to the "one-body" (open-singlet) $\Delta \mathrm{E}_{\mathrm{zz}}$ gap of Fig. 2(a), both "manybody" gaps, $\Delta \mathrm{E}_{\mathrm{zz}}$, and $\Delta \varepsilon_{\zeta \zeta}$ (the latter corresponding to the "real energetical separation of the endstates), and the one-body HOMO-LUMO gap, which involve end-states, change discontinuously at the critical length $(\sim 100 \AA)$, where $\Delta \varepsilon_{\zeta \zeta}$ and HOMO-LUMO gaps drop, while $\Delta \mathrm{E}_{\mathrm{zz}}$ increases. Thus, the observed ${ }^{8}$ gap opening (of about $0.30 \mathrm{eV}$ ) is due to the increased aromaticity at the critical 
length, and the mixing of bulk and end-states at an almost equal amount. Lawrence et $a{ }^{8}{ }^{8}$ have attributed such gap opening to the different electrostatic potential felt by valence electrons at different regions of the ribbon due to the positive partial charge on the hydrogen atoms along the sides of the AGNR. However, the paradigm of edge-modified AGNRs contradict such interpretation. ${ }^{4}$ Our present work reveals that the gap opening is a many-body effect related with the aromatic transition and the change from bulk-like $\left(\Delta \mathrm{E}_{\mathrm{ac}}\right)$ to coupled "surface-bulk" end-states $\left(\Delta \mathrm{E}_{\mathrm{zz}}\right)$. On the other hand, the calculated $\Delta \varepsilon_{\zeta \zeta}$ gap of $0.1 \mathrm{eV}$ in Fig. 2(d) is in full agreement with the results of Kimouche et al. ${ }^{13}$ Thus, Kimouche et al. ${ }^{13}$, and Lawrence et al. ${ }^{8}$, have apparently ("correctly") measured different kinds of gaps. Moreover, the same could be true for the value of $2.8 \mathrm{eV}$ measured by Zhang et al. ${ }^{12}$, which could be assigned as a tentative $\Delta \mathrm{E}_{\mathrm{ac}}$ value, either for very short AGNRs (without end-states), or for longer AGNRs with a strong "bulk" transition from deep occupied states (well below HOMO-1 orbital) to higher unoccupied states (well above the LUMO+1), and thus much larger than the real $\Delta \mathrm{E}_{\mathrm{ac}}$ (which is technically determined by the HOMO1, LUMO+1 difference). We can also observe in the 5-AGNRs that differences between the "onebody" and "many-body" (TDDFT) methods for assigning $\Delta \mathrm{E}_{\mathrm{ac}}, \Delta \mathrm{E}_{\mathrm{zz}}$, and $\Delta \varepsilon_{\zeta \zeta}$ are relatively large (or even unusual) compared to the 7- and 9-AGNRS, discussed below, where the corresponding differences are of the order of $0.1-0.2 \mathrm{eV}$. This could be related to the fact that the 5-AGNRs (contrary to 7- and 9-AGNRs) are topological and aromatic mixtures. ${ }^{3}$ Thus, the three seemingly conflicting measurements ${ }^{8,12-13}$ for the 5-AGNRs could be attributed to different length samples (and/or different positions of the STS tip). Yet, alternatively, one could claim, based on the GW results ${ }^{14}$, that there is a substrate interaction of equal magnitude $(0.85 \mathrm{eV})$ and the "real gap" is 1.7 $\mathrm{eV}$. Such conclusion is clearly considered here as highly improbable, in view of equally good (in fact better) agreement for the 7- and 9-AGNRs, not to mention Occam's principle. Moreover, if this is indeed a general trend, it clearly illustrates that elaborate correlation calculations (e.g., GW) could be avoided (see also ref. 23) if topological frustration can be taken into account appropriately by 
simple DFT (one particle) calculations, provided that the DFT functionals include "exact" exchange which is sensitive to inversion symmetry conflict. ${ }^{4}$

3.2 7-AGNRs. Figure 3 summarizes the results for the three spin states (closed singlet, open singlet, and triplet) for the 7- AGNRs, and in particular the $(7,12)$ or $3 \times 6$ AGNR. First of all, we can comment on the significance of the exact exchange in the DFT functional, which was discussed above. The calculated DFT/PBE0 open singlet $\Delta \mathrm{E}_{\mathrm{zz}}$ gap is $1.9 \mathrm{eV}$ in full agreement with the measured $^{6} \Delta \mathrm{E}_{\mathrm{zz}}$ gap for the $3 \mathrm{x} 6(7,12)$ AGNR. In contrast the $\Delta \mathrm{E}_{\mathrm{zz}}$ gap calculated with the PBE functional, which does not include "exact exchange", is less than half this value $(\sim 0.5 \mathrm{eV}$, in agreement with the PBE calculations of Wang et al. ${ }^{6}$ ). As we can see in Figs. 3(a), 3(b), and 3(c), which show the one-body DFT picture for the triplet, closed singlet, and open singlet, respectively, there are gaps in all of them between HOMO (or HOMO-1) and LUMO, which are equal or very nearly equal to the measured $\Delta \mathrm{E}_{\mathrm{zz}}$ value of $1.9 \mathrm{eV}$. We must rremember also that this value is practically equal to the correlation energy obtained from the difference between the GW and LDA values ${ }^{14}$ for these AGNRs. Let us first focus on the open singlet, which is commonly accepted as the "ground state". Figure 3(d) is practically identical with figure(s) 2(b) and 2(c) of Wang et al. ${ }^{6}$ where the definitions of $\Delta \mathrm{E}_{\mathrm{zz}}$ and $\Delta \mathrm{E}_{\mathrm{ac}}$ (which are designated as $\Delta_{\mathrm{zz}}$ and $\Delta_{\mathrm{ac}}$ respectively) are illustrated. Moreover, the calculated DFT/PBE0 $\Delta \mathrm{E}_{\mathrm{zz}}$ and $\Delta \mathrm{E}_{\mathrm{ac}}$ values (contrary to those of DFT/PBE, with no "exact exchange", used by Wang et al. ${ }^{6}$ ) are practically identical to the measured values for the $3 \times 6(7,12)$ AGNR deposited on non-metallic $\mathrm{NaCl}$ substrate (in full analogy to similar results for the 5-AGNRs, described above). Based on the closed singlet ground state, it becomes clear that the "real" energy separation of the end states is the HOMO-LUMO gap of the closed singlet state which is (almost always) about $0.1-0.3 \mathrm{eV}$ (depending on the length). This is corroborated by the TDDFT results, giving rise to the $\Delta \varepsilon_{\zeta \zeta}$ gap, which discussed earlier for the 5AGNRs, and is consistent with the appearance of Dirac points close and around the Fermi level, whereas $\Delta \mathrm{E}_{\mathrm{zz}}$ is due to mixed transitions involving both "end" and "bulk" states. 


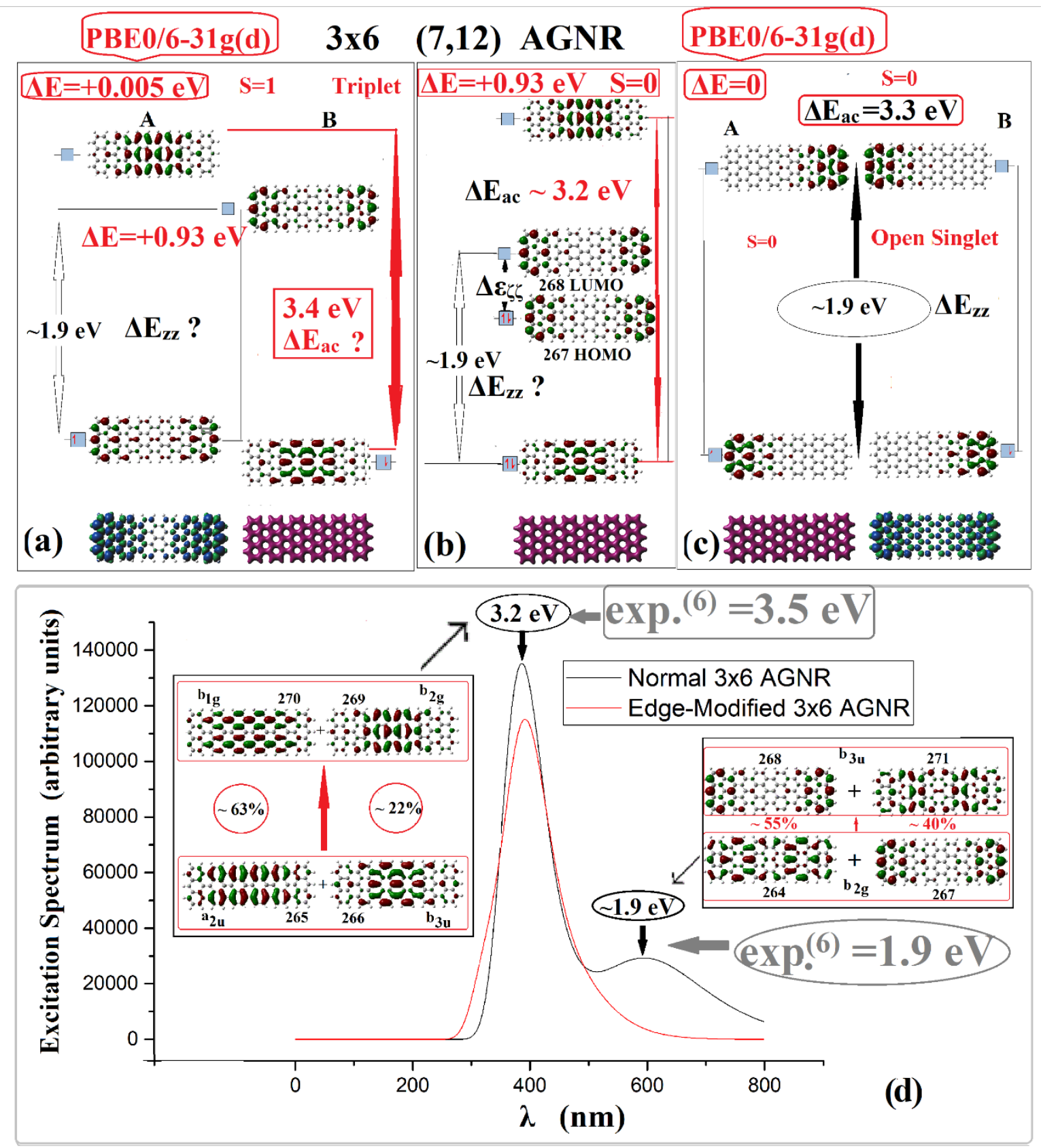

FIGURE 3. Spin states of the $3 \times 6(7,12)$ AGNR: (a) Triplet, (b) Closed Singlet, and (c) Open Singlet states, showing frontier MOs, and gaps, together with charge density and spin density (see text). (d): Excitation spectrum for the standard (black line) and edge-modified (red line on line) AGNRs. Intensity is given in arbitrary units, and excitation energy in $\mathrm{eV}$.

This is verified by Fig. 3(d) which shows the excitation spectrum of the closed singlet state for the normal 3x6 $(7,12)$ AGNR, in which there are two characteristic maxima at $1.9 \mathrm{eV}$ and $3.2 \mathrm{eV}$, which 
practically coincide with the measured $\Delta \mathrm{E}_{\mathrm{zz}}$, and $\Delta \mathrm{E}_{\mathrm{ac}}$ values respectively for this AGNR. ${ }^{6}$ As we can see in the left part of Fig. 3(d), $\Delta \mathrm{E}_{\mathrm{ac}}$ involves transitions between (mixtures of) "bulk states" (from HOMO-1 and HOMO-2, to LUMO+1 and LUMO+2), whereas $\Delta \mathrm{E}_{\mathrm{zz}}$ corresponds to transitions from mixt, "bulk" + "surface" (HOMO-3 and HOMO) to LUMO+3 and LUMO. Thus, $\Delta \mathrm{E}_{\mathrm{zz}}$, although not equal to energy separation of the end states, is clearly associated with the first (lowest energy) transition involving end and bulk states, corresponding to the measured $\Delta \mathrm{E}_{\mathrm{zz}}$ value of $1.9 \mathrm{eV}$ and the magnitude of the open singlet gap. This is also supported by the TDDFT results in Fig. 3(d) showing the spectrum of the edge-modified closed singlet in which the peak of $1.9 \mathrm{eV}$ is totally absent, whereas the peak of the "bulk" gap $\Delta \mathrm{E}_{\mathrm{ac}}$ is identical to the $3.2 \mathrm{eV}$ peak of the normal $(7,12)$ AGNR. The position of the $\Delta \mathrm{E}_{\mathrm{ac}}$ peak, contrary to $\Delta \mathrm{E}_{\mathrm{zz}}$, changes (decreases) as the length increases. Thus, for the $3 \times 14(7,28)$ AGNR we found a $\Delta \mathrm{E}_{\mathrm{ac}}$ value of $2.8 \mathrm{eV}$, as is shown in Fig. S2(a). This value of $2.8 \mathrm{eV}$, as could be expected, is in perfect agreement with the calculated GW value ${ }^{6}$ and the experimental measurements for the $(7,24-28)$ AGNR(s) on insulating $\mathrm{NaCl}$ substrate. ${ }^{6}$

3.3 9-AGNRs. We can observe in Fig. S2(c) that the overall spectrum of the 4x6 AGNR which has the same length with the 3x6 AGNR, except for a suppression of the $\Delta \varepsilon_{\zeta \zeta}$ peak, looks at a first sight very much alike the one for the 3x6 AGNR. Clearly a (deep) "bulk" gap could be expected not to vary very much or be sensitive to the exact AGNR's width; but for the peak around $2.0 \mathrm{eV}$, which up to now was associated with the $\Delta \mathrm{E}_{\mathrm{zz}}$ gap of the $3 \mathrm{x}-\mathrm{AGNRs}$, further investigation is needed, which is described in Fig. 4. Figures 4(a), 4(b), and 4(c) are the corresponding analogues of figures 3(a), 3(b), and 3(c) respectively. However, contrary to the 7-AGNRs, the experimental data for the 9-AGNRs are very limited. ${ }^{16}$ Therefore most of the results shown in Fig. 4 should be considered as predictions of the present work. As we can see in Fig. 4(c) for the open singlet the two fundamental gaps $\Delta \mathrm{E}_{\mathrm{zz}}$ and $\Delta \mathrm{E}_{\mathrm{ac}}$ are very close together (2.2 eV and $2.4 \mathrm{eV}$ respectively) and not exactly equal to the corresponding $3 \times 6$ gaps This is also true for the almost equal values of $\Delta \mathrm{E}_{\mathrm{zz}}$ and $\Delta \mathrm{E}_{\mathrm{ac}}$ obtained from triplet (and closed singlet). Thus, the peak around $2.0 \mathrm{eV}$ in Fig. $4(\mathrm{~d})$ is the 
result of the overlap of the $\Delta \mathrm{E}_{\mathrm{zz}}$ and $\Delta \mathrm{E}_{\mathrm{ac}}$ gaps, whereas the peak around $3.2 \mathrm{eV}$ in the same figure, Fig. 4(d), although of "bulk" type (similarly to the 3x6 AGNR) is not the smallest "bulk" gap, and the real $\Delta \mathrm{E}_{\mathrm{ac}}$ for the $4 \mathrm{x} 6(9,12) \mathrm{AGNR}$ should be around $2.0 \mathrm{eV}$.
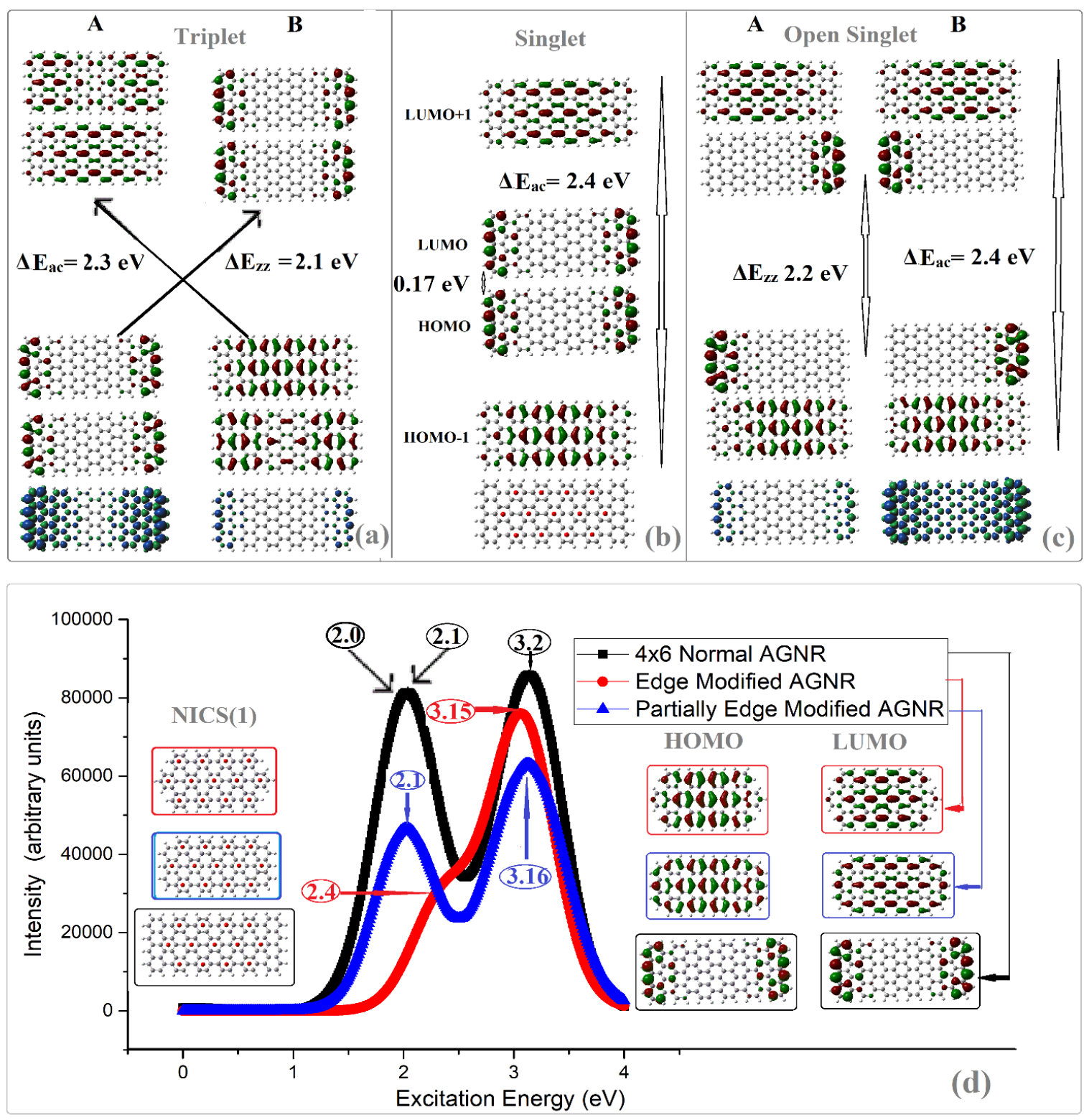

FIGURE 4. Spin states of the 4x6 $(9,12)$ AGNR: (a) Triplet, (b) Closed Singlet, and (c) Open Singlet, showing frontier MOs, gaps, and spin densities; (d)Excitation spectrum for the standard (black line), partially edge-modified (blue line on line), and fully edge modified AGNRs (red line on line). Intensity is given in arbitrary units, and excitation energy in $\mathrm{eV}$. The frontier orbitals and 
aromaticity patterns of partially and fully edge-modified AGNRs are shown in the right and left portions of the figure.

This is verified in Fig. 4(d), which shows that the $2.1 \mathrm{eV}$ "bulk" peak (together with the "deeper" 3.2 $\mathrm{eV}$ "bulk" peak) survives the elimination (total and partial) of the empty (non-aromatic) end-rings which generates the edge modified AGNRs (without end states, and $\Delta \mathrm{E}_{\mathrm{zz}}$ ). As is well known, this "bulk" peak value, decreases as the length of the AGNR increases. For the $4 \times 13$ AGNR we find $\Delta \mathrm{E}_{\mathrm{ac}}$ $=1.6 \mathrm{eV}$, but for the longer $4 \times 18(9,36)$, and $4 \times 24(9,48)$ AGNRs of lengths $\mathrm{L} \approx 78 \AA$, and $\mathrm{L} \approx 104 \AA$ respectively, we obtain (by TDDFT) for both of them $\Delta \mathrm{E}_{\mathrm{ac}}=1.45 \mathrm{eV}$. This value is in very good agreement with the recently measured gap of $1.4 \mathrm{eV}$ by Talirz et al. ${ }^{16}$, as is illustrated in Fig. S3. The peak at $1.45 \mathrm{eV}$ is further verified by Fig. $\mathrm{S} 4$ which shows the spectrum of the edge-modified $4 \times 24$ AGNR. We can also clearly see in Figs. S3(b), S3(c) the "surface" $\Delta \mathrm{E}_{\mathrm{zz}}$ gap at about 2.1-2.2 eV. Thus, for the 9-AGNRs the predicted values for the gaps are $\Delta \mathrm{E}_{\mathrm{ac}}=1.45 \pm 0.1 \mathrm{eV}$, and $\Delta \mathrm{E}_{\mathrm{zz}}=2.1 \pm 0.1$ $\mathrm{eV}$.

4. Conclusions. We have achieved an excellent agreement (within $1 \%$ or less) with, and identification / rationalization of the measured STS gaps ("bulk" and "surface") for the known 5-, 7- and 9-AGNRs. Namely:

a) For the 5-AGNRs the measured ${ }^{8}$ gap value is $0.85 \mathrm{eV}$. The calculated here gap with DFT/PBE0 is 1.07 , whereas the TDDFT/PBE0 value is exactly $0.85 \mathrm{eV}$, indicating also that this is a $\Delta \mathrm{E}_{\mathrm{zz}}$ gap.

b) Moreover, the measured ${ }^{13} 0.1 \mathrm{eV}$ gap is recognized to fully coincide with the calculated here (by both DFT-TDDFT/PBE0) $\Delta \varepsilon_{\zeta \zeta}$ gap.

c) For the 7-AGNRs the measured ${ }^{6,7,9,10,15} \Delta \mathrm{E}_{\mathrm{ac}}$ gap of $2.3 \pm 0.2 \mathrm{eV}$ coincides with the calculated here $\Delta \mathrm{E}_{\mathrm{ac}}$ gap (with both DFT-TDDFT/PBE). 
d) Furthermore, for the $(7,28)$ AGNR the measured ${ }^{6}$ and GW-calculated ${ }^{6} 2.8 \mathrm{eV}_{\text {gap fully }}$ coincides with the calculated here $\Delta \mathrm{E}_{\mathrm{ac}}$ gap (with both DFT-TDDFT/PBE0), whereas for the $(7,12)$ AGNR the measured ${ }^{6}$ and calculated ${ }^{6} \Delta \mathrm{E}_{\mathrm{ac}}$ gap is $\sim 3.2 \mathrm{eV}$.

e) The measured ${ }^{6} \Delta \mathrm{E}_{\mathrm{zz}}$ gap of $1.9 \mathrm{eV}$ for the 7-AGNRs, $(7,12)$, and longer, is clearly identical to the calculated here $\Delta \mathrm{E}_{\mathrm{zz}}$ gap of $1.9 \mathrm{eV}$ (with both DFT-TDDFT/PBE0).

f) For the 9-AGNRs the only known (to the present author) measurement ${ }^{16}$ for the gap is 1.4 $\mathrm{eV}$. The present calculations (TDDFT/PBE0) yield a $\Delta \mathrm{E}_{\mathrm{ac}}$ value of $1.45 \mathrm{eV}$, and also predict $\Delta \mathrm{E}_{\mathrm{zz}}=2.1 \pm 01 \mathrm{eV}$, quite close to the corresponding gap for the 7-AGNRs.

These results, which have been obtained at the lowest possible computational cost and maximum physical insight, using a simple, transparent, but highly efficient process, imply:

1) minimum substrate contribution to the measured STS gaps

2) maximum Coulomb correlation of almost equal magnitude with the staggered potential,

Table 1 summarizes these results and conclusions.

TABLE 1. Calculated and measured gaps for the 5-, 7-, and 9-AGNRs (in eV). Numbers with asterisk denote the present values, while numbers in parenthesis indicate the reference numbers of the original works. Numbers in bold emphasize the agreement between theoretical and experimental results, whereas underlined numbers in italics indicated the results of GW calculations. ${ }^{14}$

\begin{tabular}{|c|c|c|c|c|c|c|}
\hline AGNR & $\begin{array}{c}\Delta \varepsilon_{\zeta \zeta} \\
\text { Calculated }\end{array}$ & $\begin{array}{c}\Delta \varepsilon_{\zeta \zeta} \\
\text { Measured }\end{array}$ & $\begin{array}{c}\Delta \mathbf{E}_{\mathrm{zz}} \\
\text { Calculated }\end{array}$ & $\begin{array}{l}\Delta \mathrm{E}_{\mathrm{zz}} \\
\text { Measured }\end{array}$ & $\begin{array}{l}\Delta \mathbf{E}_{\mathrm{ac}} \\
\text { Calculated }\end{array}$ & $\begin{array}{l}\Delta \mathbf{E}_{\mathbf{a c}} \\
\text { Measured }\end{array}$ \\
\hline $5-$ & $0.1^{*}(1)$ & $0.1^{(13)}$ & $0.85^{*}$ & $\mathbf{0 . 8 5}^{(8)}$ & $1.1^{*},{\underline{1.7^{(14)}}}^{(14)}$ & - \\
\hline 7- & $0.1^{*(1)}$ & - & $1.9^{*}$ & $\mathbf{1 . 9 ^ { ( 6 ) }}, 2.5^{(7)}$ & $2.5^{*}, 2.8^{*}, 2.8^{(6)}, \underline{3.7}^{(14)}$ & $\mathbf{2 . 8 ^ { ( 6 ) }}, \mathbf{2 . 5} \pm 0.2^{(6,7,9,15}$ \\
\hline 9- & $0.1^{*(1)}$ & - & $2.2^{*}$ & - & $1.45^{*}, 1.6^{(1)}, 2.0^{(14)}$ & $1.4^{(16)}$ \\
\hline
\end{tabular}

*values obtained in the present work.

Additional supplementary material is given in the Supplementary Information. 


\section{References}

1. Zdetsis, A. D.; and Economou, E.N. Rationalizing and Reconciling Energy Gaps and Quantum Confinement in Narrow Atomically Precise Armchair Graphene Nanoribbons. Carbon 2017, 116, 422-434.

2. Zdetsis, A. D. Bridging the Physics and Chemistry of Graphene(s): From Hückel's Aromaticity to Dirac's Cones and Topological Insulators. J. Phys. Chem. A 2020, 124, 976-986

3. Zdetsis, A. D. Do We Really Understand Graphene Nanoribbons? A New Understanding of the 3n, 3n \pm 1 Rule, Edge "Magnetism” and Much More. J. Phys. Chem. C 2020, 124, 7578-7584

4. Zdetsis A. D.; Economou, E. N. Topological Metal-Insulator Transition in Narrow Graphene Nanoribbons? Carbon 2021, 176, 548-557

5. Zdetsis, A. D. $4 n+2=6 n$ ? A Geometrical Approach to Aromaticity? J. Phys. Chem. A 2021, 125, 6064-6074

6. Wang, S. et al. Giant edge state splitting at atomically precise graphene zigzag edges. Nat. Commun. 20167 : 1150. DOI: 10.1038/ncomms 115077

7. Kolmer, M. et al. Rational synthesis of atomically precise graphene nanoribbons directly on metal oxide surfaces. Science 2020, 369, 571-575DOI: 10.1126/science.abb8880

8. Lawrence, J. et al. Probing the Magnetism of Topological End States in 5-Armchair Graphene Nanoribbons. ACS Nano 2020, 14, 4499-4508

9. Chen, Y-C; Oteyza, D. G.; Pedramrazi, Z; Chen, C.; Fischer, F. R.; Crommie, M. F. Tuning the Band Gap of Graphene Nanoribbons Synthesized from Molecular Precursors. ACS Nano. 2013, $6123-6128$.

10. Song, S. et al. On-surface synthesis of graphene nanostructures with $\pi$-magnetism Chem. Soc. Rev. 2021, 50, 3238-3262

11. Nakada K.; Fujita M.; Dresselhaus G.; Dresselhaus M. S. Edge state in graphene ribbons: Nanometer size effect and edge shape dependence. Phys. Rev. B 1996 54, 17954 
12. Zhang, H.; Lin, H; Sun, K. et al. On-surface synthesis of rylene-type graphene nanoribbons. J. Am. Chem. Soc. 2015 137, 4022-4025.

13. Kimouche, A; Ervasti, M. M.; Drost, R.; Halonen, S.; Harju, A.; Joensuu, P. M.; Sainio, J.; and Liljeroth, P. Ultra-narrow metallic armchair graphene nanoribbons. Nature Comm. 2015, 6:10177, $1-6$.

14. Yang, L.; Park, C.-H.; Son, Y.-W.; Cohen, M. L.; Louie, S. G. Quasiparticle energies and band gaps in graphene nanoribbons. Phys. Rev. Lett. 2007, 99, 186801

15. Ruffieux, P.; Cai, J.; Plumb, N. C.; Patthey, L.; Prezzi, D.; Ferretti, A.; Molinari, E.; Feng, X.; Müllen, K.; Pignedoli, C. A.; et al. Electronic Structure of Atomically Precise Graphene Nanoribbons. ACS Nano 2012, 6, 6930-6935.

16. Talirz, L.; Söde, H; Dumslaff, T. et al. On-Surface Synthesis and Characterization of 9-Atom Wide Armchair Graphene Nanoribbons. ACS Nano. 2017, 11, 1380-1388.

17. Overbeck, J. et al. A Universal Length-Dependent Vibrational Mode in Graphene Nanoribbons. ACS Nano 2019, 13, 13083-13091

18. Varykhalov, A.; Sánchez-Barriga, J.; Shikin, A. M.; Biswas, C.; Vescovo, E.; Rybkin, A. ; D. Marchenko, D.; Rader, O. Electronic and Magnetic Properties of Quasifreestanding Graphene on Ni. Phys. Rev. Lett. 2008, 101, 157601.

19. Zdetsis. A. D. The real structure of the $\mathrm{Si}_{6}$ cluster, Phys. Rev. A 2001, 64, 23202

20. Son, Y.-W. Cohen, M. L.; Louie, S. G. Energy Gaps in Graphene Nanoribbons. Phys. Rev. Lett. 2006, 97, 216803

21. Ijäs, M. et al. Electronic states in finite graphene nanoribbons: Effect of charging and defects. Phys. Rev. B 2013, 88, 07542976

22. Frisch, M. J. et al. Gaussian 09, revision C.01, Gaussian, Inc.: Wallingford, CT, 2009.

23. GaussView, version 5.09, Dennington, R.; Keith, T.; Millam, J. Semichem Inc.: Shawnee Mission, KS, 2016. 
24. Zdetsis, A. D.; Niaz, S. From Zero to Infinity: Customized Atomistic Calculations for Crystalline Solids -Applications to Graphene and Diamond. Adv. Mater. Lett. 2021, 12(9), 21091659. DOI:10.5185/amlett.2021.091659 